\title{
UGC 7639: A Dwarf Galaxy in the Canes Venatici I Cloud
}

\author{
L. M. Buson, ${ }^{1}$ D. Bettoni, ${ }^{1}$ P. Mazzei, ${ }^{1}$ and G. Galletta ${ }^{2}$ \\ ${ }^{1}$ INAF Osservatorio Astronomico di Padova, Vicolo dell'Osservatorio 5, 35122 Padova, Italy \\ ${ }^{2}$ Dipartimento di Fisica e Astronomia, Vicolo dell'Osservatorio 2, 35122 Padova, Italy \\ Correspondence should be addressed to L. M. Buson; lucio.buson@oapd.inaf.it
}

Received 24 April 2015; Revised 17 June 2015; Accepted 1 July 2015

Academic Editor: Duncan Forbes

Copyright (C) 2015 L. M. Buson et al. This is an open access article distributed under the Creative Commons Attribution License, which permits unrestricted use, distribution, and reproduction in any medium, provided the original work is properly cited.

\begin{abstract}
We want to get insight into the formation mechanism and the evolution of UGC 7639, a dwarf galaxy in the Canes Venatici I Cloud (CVnIC). We used archival multiwavelength data to constrain its global properties. Ultraviolet images show that UGC 7639 inner regions are composed mostly by young stellar populations. In addition, we used smoothed particle hydrodynamics simulations with chemophotometric implementation to account for its formation and evolution. UGC 7639 is an example of blue dwarf galaxy whose global properties are well matched by our multiwavelength approach, that is, a suitable approach to highlight the evolution also of these galaxies as a class. We found that the global properties of UGC 7639, namely, its total absolute B-band magnitude, its whole spectral energy distribution, and morphology, are well matched by an encounter with a system four times more massive than our target. Moreover, the current star formation rate of the simulated dwarf, $\approx 0.03 \mathrm{M}_{\odot} \mathrm{yr}^{-1}$, is in good agreement with our UV-based estimate. We derived a galaxy age of 8.6 Gyr. Following our simulation, the ongoing star formation will extinguish within $1.6 \mathrm{Gyr}$, thus leaving a red dwarf galaxy.
\end{abstract}

\section{Introduction}

Dwarf galaxies (DGs) are the predominant class in the total galaxy population of the universe. They are, by definition, galaxies of low mass and size and could be considered as the smallest baryonic counterparts of the dark matter (DM) constituent blocks in the universe [1-3]. Despite their abundance, we can study in detail only the objects that we see in the local universe, due to their intrinsic faintness. In particular, in the nearby Local Volume (LV), the most detailed information available about DGs comes from the LV population (cf. $[4,5])$. However, many recent studies have started to look beyond the LV in order to derive the properties of DGs belonging to several nearby groups. These studies include Cen A, NGC 1407, Coma I, Leo, NGC 1023, M81, Sculptor, and Canes Venatici Cloud itself (e.g., [5-9]).

DGs possess a large variety of morphological types [10], the most frequent in galaxy groups being dwarf elliptical (dE), dwarf spheroidal (dSph), and dwarf irregular (dIrr) galaxies $[10,11]$.

Among them, dIrrs are galaxies showing recent star formation, exhibiting substantial gas fractions, and a broad range of star formation rates (SFR). The majority of dIrrs belong to groups rather than being found in the field [12].

The evolutionary properties of dwarf galaxies in nearby groups are still not well understood. Their study can help to understand the role of the environment and interactions on galaxy evolution. Moreover, these galaxies can reasonably be considered local analogs of the farthest (i.e., youngest) actively star-forming systems.

Our goal is to investigate the evolution of a specific dIrr in a group, UGC 7639. This is a dwarf galaxy belonging to the relatively large number of nearby low surface brightness galaxies investigated in the context of the recent imaging surveys of the LV neighborhood. The distance of UGC 7639 has been reliably estimated by means of the surface brightness fluctuation method by Rekola et al. [13]. They estimated $D=$ $7.1 \pm 0.6 \mathrm{Mpc}$, in excellent agreement with the value (7.14 \pm $0.5 \mathrm{Mpc}$ ) given in the updated nearby galaxy catalogue of Karachentsev et al. [14]. UGC 7639 is a member of the Canes Venatici Cloud [15]. The so-called Canes Venatici Cloud consists of two complexes aligned along the same line of sight, including mainly late-type DGs. More specifically, it is formed by CVnI Cloud and CVnII Cloud, at $V_{r}=300 \mathrm{~km} \mathrm{~s}^{-1}$, 


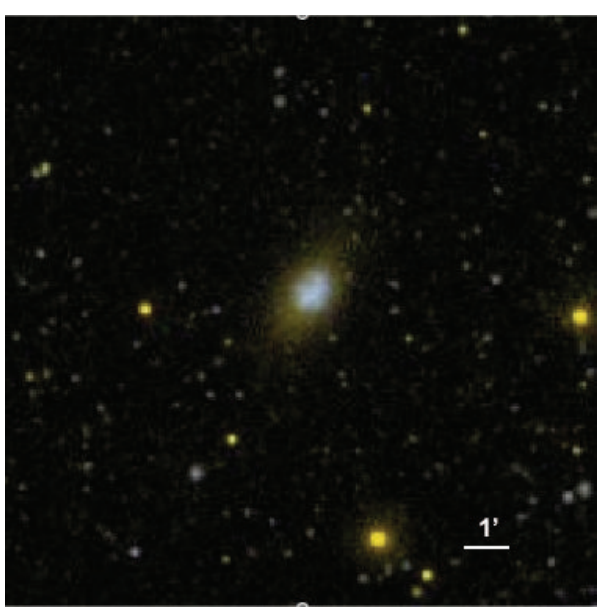

(a)

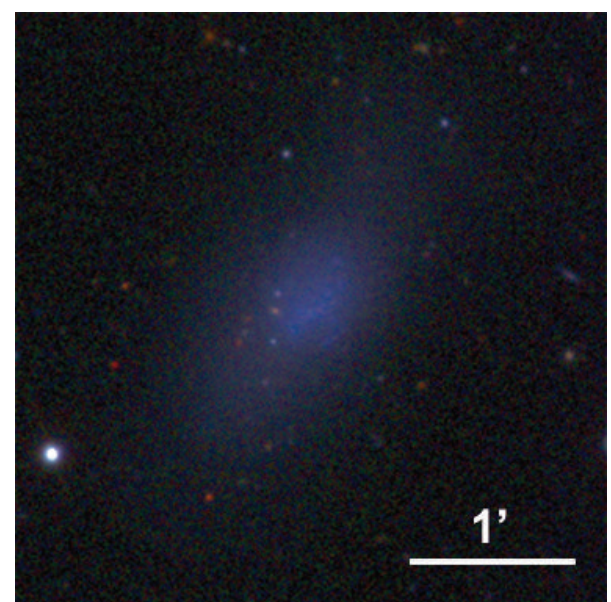

(b)

FIgURE 1: (a) Composite $13^{\prime} \times 13^{\prime}$ GALEX image of UGC 7639. Blue and yellow correspond to FUV and NUV. (b) Composite $3^{\prime} .6 \times 3^{\prime} .6$ SDSS (gri) image of UGC 7639. Note the outstanding, innermost blue patches, which are also weak $\mathrm{H} \alpha$ emitters.

that is, at an average distance of $7.68 \pm 0.9 \mathrm{Mpc}$, and $V_{r}=$ $560 \mathrm{~km} \mathrm{~s}^{-1}$, that is, at an average distance of $17.2 \pm 1.3 \mathrm{Mpc}$, respectively (cf. [16]). As a consequence, UGC 7639, with a measured redshift of $382 \mathrm{~km} \mathrm{~s}^{-1}$ [13], likely belongs to the CVnI Cloud.

UGC 7639 caught our attention because its central regions, as recorded by the GALEX satellite, appear dominated by FUV emission (Figure 1(a)). This object likely hosts an amount of young stars typical of an ongoing starburst. This is also a general property of DGs of different morphological types ([10, 17]; e.g., [18]).

In the past, several different morphological classifications have been proposed for this object. deVaucouleurs et al. [19] (RC3) and later Rekola et al. [13] proposed a transition class $\mathrm{dE} / \mathrm{Im}$. Conversely, a dS0/BCD (Blue Compact Dwarf) classification has been adopted by Bremnes et al. [20] and Parodi and Binggeli [21] considering its outer diffuse elliptical halo and inner multiple star-forming regions and star clusters. Finally, Karachentsev et al. [14] favor a true Im classification. Indeed, the galaxy shows a pattern of inner star-forming regions easily seen in the SDSS composite image (Figure $1(\mathrm{~b})$ ), although the $\mathrm{H} \alpha$ imaging by Kaisin and Karachentsev [22] barely supports this last classification.

In this paper, we want to investigate the formation mechanism and the evolution of this dwarf galaxy, as an example of DG evolution.

We used GALEX (FUV and NUV) and SDSS $g$ and $r-$ band images as well as Hyperleda and NED data to constrain its global properties. In addition, smoothed particle hydrodynamics (SPH) simulations with chemophotometric implementation gave us insight into its formation mechanism, together with the past and future evolution of this galaxy. These SPH simulations allowed us to derive dynamical and morphological information, concurrently with the spectral energy distribution (SED) extended from far-UV to $1 \mathrm{~mm}$, at each evolutionary time (snapshot, hereafter). Finally, they allowed us to trace the evolutionary path of the selected galaxy in the optical and UV color-magnitude diagram, (NUV-r) versus $M_{r}$.

The present work is organized as follows: in Section 2, archive multiwavelength data are analyzed and physical properties of our target are derived. The UV-optical colormagnitude diagram (CMD) of all 22 members of the Canes Venatici I Cloud, to which our target belongs, is also examined to characterize the surrounding environment. Section 3 focuses on the description of the adopted modeling and on our results. Finally, conclusions are summarized and discussed in Section 4.

The selected dwarf, with a reliable distance measured, a large wavelength coverage of the observed SED (Section 3), and other properties discussed in the following section (Section 2), provides an useful example of this class of objects, helping us to constrain our simulations and giving insights into the formation mechanism and the evolution of this dwarf as an example of DG evolution.

\section{Physical Parameters}

2.1. UV/Optical Photometry. To study both the UV and the optical morphology, we extracted two UGC 7639 images ( $3249 \mathrm{~s}$ and $5883 \mathrm{~s}$ for FUV and NUV cameras, resp.) from the GALEX archive (they belong to tile G11_47072_UGC07639, obtained as part of the program GI1 047 P.I. R. Kennicutt) together with $\mathrm{r}$ - and g-band images belonging to the SDSSDR7 archive [23].

From these images we performed the surface photometry by means of the IRAF STSDAS ELLIPSE routine for NUV, FUV, and $g$ and $r$ bands. ELLIPSE computes a Fourier expansion for nested isophotes [24], producing the surface photometric profiles. The resulting UV photometric profiles and corresponding (FUV-NUV), (NUV-r) color profiles versus circularized radius are shown in Figure 2. The circularized radius has been derived from the relation $r_{c}=a_{e}{ }^{*}(1-\varepsilon)^{1 / 2}$, where $a_{e}$ is the radius measured along the ellipse major axis and $\varepsilon$ is the measured galaxy ellipticity. The average colors 


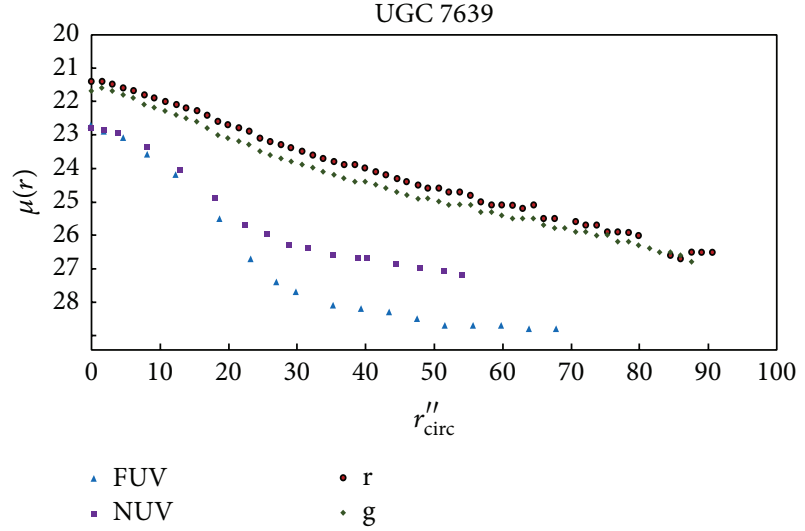

(a)

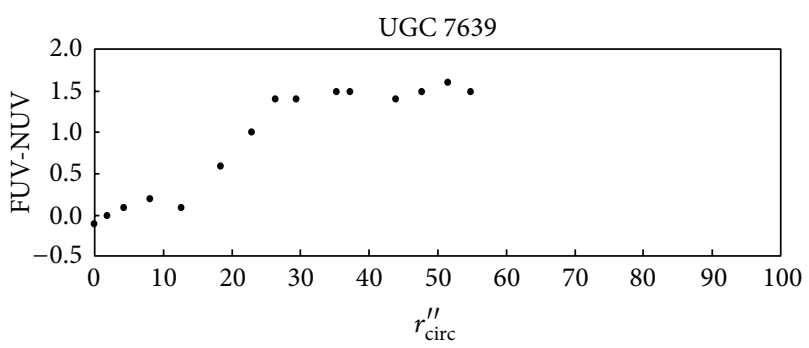

(b)

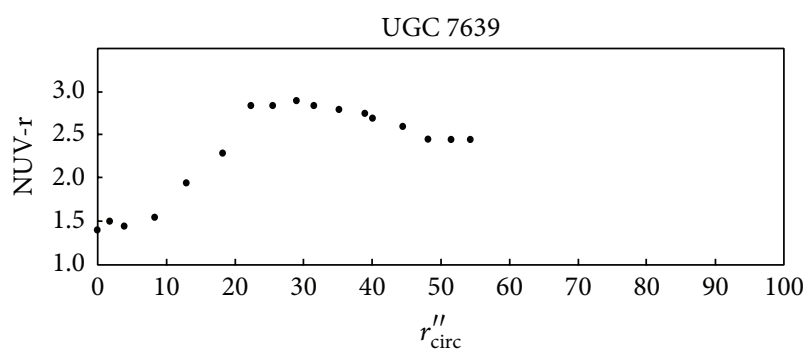

(c)

Figure 2: (a) GALEX NUV and FUV and optical r- and g-band surface brightness profiles of UGC 7639 (magenta squares, cyan triangles, and red and green circles, resp.) versus circularized radius. (b) (FUV-NUV) color profile. (c) UV/optical (NUV-r) color profile. Since BCDs experience mostly off-centered star formation (cf. [18]), these profiles could be affected by the difficult identification of the main stellar body centre.

(derived inside the full extent of the galaxy in the GALEX images: $\sim 60)$ are (FUV-NUV) $=0.40 \pm 0.07$ and $($ NUV-r $)=$ $2.58 \pm 0.18$ which are typical of late type DGs [25]. However in its innermost region (10 arcsecs, i.e., $\approx 0.4 \mathrm{kpc}$ ), the galaxy shows much bluer colors; that is, $(\mathrm{FUV}-\mathrm{NUV})=0.06 \pm 0.11$ and $(\mathrm{NUV}-\mathrm{r})=1.47 \pm 0.06$ (Figure $2(\mathrm{c})$ ) a characteristic of dIrrs [26].

Our resulting $\mathrm{g}$ and $\mathrm{r}$ magnitudes, effective radii, and average surface brightness are reported in Table 1. Taking into account the classical Fukugita et al. [27] conversion formulae, we compared our integrated $\mathrm{g}$ and $\mathrm{r} \mathrm{AB}$ magnitudes with those of Bremnes et al. [20] in the Johnson B and R bands, that is, $m_{B}=13.94 \pm 0.21$ and $m_{R}=12.93 \pm 0.20$ mag, finding a good agreement.
TABLE 1: Measured optical photometric parameters.

\begin{tabular}{lcccc}
\hline Band & $\begin{array}{c}r_{e} \\
\operatorname{arcsec}\end{array}$ & $\begin{array}{c}\mu_{e} \\
\operatorname{mag~arcsec}^{-2}\end{array}$ & $\begin{array}{c}m_{\text {tot }} \\
\text { mag }\end{array}$ & $\begin{array}{c}M_{\text {tot }} \\
\text { mag }\end{array}$ \\
\hline $\mathrm{r}[\mathrm{AB}]$ & 30 & 22.9 & $13.12 \pm 0.08$ & -16.14 \\
$\mathrm{~g}[\mathrm{AB}]$ & 31 & 23.6 & $13.51 \pm 0.10$ & -15.75 \\
\hline
\end{tabular}

We found that the best agreement with the observed $g$ and $r$ profiles is obtained with a pure disk component. The FUV and NUV profiles show a light excess in the central region, due to UV spots (see Figure 1), that cannot be fitted with a smooth component. For this reason we give here only the total magnitudes, NUV $=15.7 \pm 0.04$ and $\mathrm{FUV}=16.1 \pm 0.04$. These integrated magnitudes, in good agreement with the values NUV $=15.71 \pm 0.03$ and FUV $=16.13 \pm 0.05$ measured by Lee et al. [28], will be used to constrain our simulations (Section 3.1.1).

2.2. The Environment. As already pointed out, UGC 7639 belongs to the CVnI Cloud that includes 22 galaxies [15]. To characterize UGC 7639 inside this Cloud we obtained, for each galaxy, the available SDSS r-band magnitudes from the NASA-Sloan Atlas [29] and the NUV and FUV data from Lee et al. [28]. Extinction corrections have been extracted, for each galaxy, from the same NASA-Sloan Atlas [29].

For UGC 7639 we used the updated distance modulus by Rekola et al. [13], while for the other galaxies we used the distances given by Tully [15]. Using these data, we derive the color-magnitude diagram (CMD) (NUV-r) versus $M_{r}$ $[25,30,31]$, shown in Figure 3. All but four galaxies follow the blue sequence (cyan line), and no galaxy lies on the red sequence, typical of evolved and/or reddened systems. NGC 4096, NGC 4248, NGC 4460, and NGC 4707 are in the intermediate region, the so-called green valley. Following the classification of Tolstoy et al. [4], there are eight DGs (i.e., $M_{B}>-16.0 \mathrm{mag}$ ) in this Cloud, accounting for $\sim 40 \%$ of its member galaxies, and looking at Figure 3, UGC 7639 is among the faintest members of this group.

The UV luminosity is a tracer of the present-day SFR that can be derived following Kennicutt Jr. [32], from the relation

$$
\operatorname{SFR}_{\mathrm{FUV}}\left(\mathrm{M}_{\odot} \mathrm{yr}^{-1}\right)=1.4 \times 10^{-28} L_{\mathrm{FUV}}\left(\operatorname{ergs~s}^{-1} \mathrm{~Hz}^{-1}\right) \text {. }
$$

Using the $L_{\mathrm{FUV}}$ from FUV magnitudes that we measured (Section 2.1) and our adopted distance, the SFR turns out to be $\sim 10^{-2} \mathrm{M}_{\odot} \mathrm{yr}^{-1}$ for our dwarf, in agreement with the value found by Karachentsev and Kaisina [33]. Such relation accounts for a Salpeter's IMF with lower and upper mass limits $0.1 \mathrm{M}_{\odot}$ and $100 \mathrm{M}_{\odot}$, respectively. The value of the SFR here derived will be used to further constrain our simulation (Section 3.1.1).

Since total UV magnitudes are available for each member of the Cloud [28], using the previous formula we highlight with different symbols in Figure 3 the different levels of the present-day SFR of the galaxies in this Cloud. Only few members harbor a SFR above $0.1 \mathrm{M}_{\odot} / \mathrm{yr}$ (squares). NGC 4258, the brightest galaxy in such figure, reaches the highest value, namely, $0.6 \mathrm{M}_{\odot} / \mathrm{yr}$. The Tully [15] catalog also provides the 


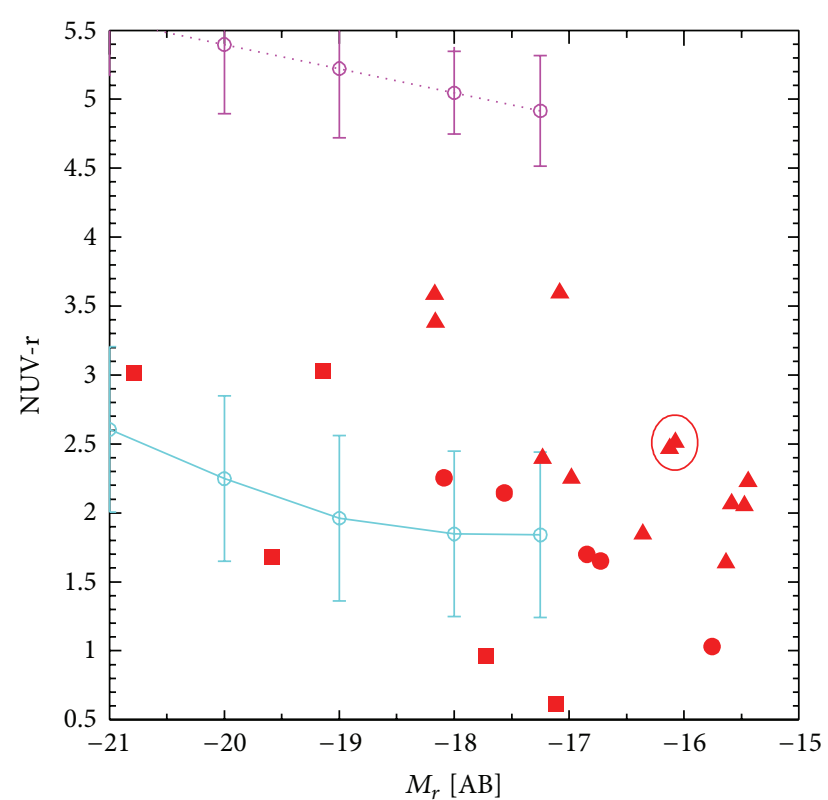

Figure 3: The CMD (NUV-r) versus $M_{r}$ of the CVnI Cloud members. The Wyder et al. [25] fits of the blue and red sequences (cyan/magenta lines) are plotted, including their error bars. The oval indicating the position of UGC 7639 also includes UGC 7408, a galaxy slightly bluer and brighter than our dwarf. Different symbols correspond to different SFRs: squares are for SFR $\geq 0.1 \mathrm{M}_{\odot} / \mathrm{yr}$, triangles for SFR $\leq 0.05 \mathrm{M}_{\odot} / \mathrm{yr}$, and circles for SFRs in between.

amount of cold gas (HI) in 19 out of 22 galaxies in the Cloud. We complete our sample by adding data of NGC 3985 and NGC 4460 from Springob et al. [34] and of NGC 4485 from the RC3 catalog, respectively. UGC 7639 contains the lowest amount of neutral gas $\left(\sim 4 \times 10^{7} \mathrm{M}_{\odot}\right)$ in the Cloud, while NGC 3985 contains the highest amount, namely, $\sim 10^{11} \mathrm{M}_{\odot}$. The amount of cold gas in our DG constrained further our simulations (Section 3.1.1).

\section{Modeling}

The novelty of our approach is that we explore the evolutionary scenario of our selected galaxy using a large set of SPH simulations of galaxy encounters and/or mergers, including a chemophotometric code based on evolutionary population synthesis (EPS) models. The general prescriptions of SPH simulations and the grid of impact parameter explored, reported in several previous papers (e.g., [35, 36]), are summarized below.

Our SPH simulations of galaxy formation and evolution start from the same initial conditions described in Mazzei and Curir ([37], MC03 hereafter) and Mazzei ([38] and references therein), that is, collapsing triaxial systems composed of DM and gas with density distribution $\rho \propto r^{-1}$, in different proportions and different total masses. All of the simulated systems have the same initial virial ratio (0.1), as well as the same average density and spin parameter. In more detail, each system is built up with a spin parameter, $\lambda$, given by $|J| \mid E^{0.5} /\left(G M^{0.5}\right)$, where $E$ is the total energy, $J$ is the total angular momentum, and $G$ is the gravitational constant; $\lambda$ is equal to 0.06 and is aligned with the shortest principal axis of the DM halo. The triaxiality ratio of the DM halo, $\tau=\left(a^{2}-b^{2}\right) /\left(a^{2}-c^{2}\right)$, is 0.84 , where $a>b>c$ [39].

We then produced a large set of galaxy encounters involving systems with a range of mass ratios from 1:1 to $1: 10$. In order to exploit the vast range of orbital parameters, we carried out different simulations for each pair of interacting systems, varying the orbital initial conditions in order to have, for an ideal Keplerian orbit of two mass points, the first pericentre separation $(p)$ ranging from the initial length of the major axis of the DM triaxial halo of the primary system to $1 / 10$ of the same (major) axis. For each of these separations, we changed the eccentricity in order to have hyperbolic orbits of different energies. For the most part, we studied direct encounters, where the spins of the systems are equal (MC03), generally parallel to each other, and perpendicular to the orbital plane. However, we also analyzed some cases with misaligned spins in order to enhance the effects of the system initial rotation on the results. Moreover, for a given set of encounters with the same orbital parameters, we also examined the role of increasing initial gas fractions.

All of the simulations include the self-gravity of gas, stars and DM, radiative cooling, hydrodynamical pressure, shock heating, viscosity, star formation, feedback from evolving stars and type II supernovae, and chemical enrichment. Simulations provide the SED at each evolutionary time, that is, at each snapshot. The time step between individual snapshots is 0.037 or $0.151 \mathrm{Gyr}$, as for the simulation which best fits the global properties of UGC 7639 (see below). The SED we derived accounts for chemical evolution, internal extinction, and reemission by dust in a self-consistent way and extends at least over four orders of magnitude in wavelength, that is, from 0.06 to $1000 \mu \mathrm{m}$. The SED is based on EPS models exploiting isochrones, and this method has been fully described in several previous papers [40-42]. Paper [40] by Mazzei et al. in particular was the first paper where the SED is extended over four orders of magnitude in wavelength [43]. SPH simulations with chemophotometric implementation were presented for the first time by Curir and Mazzei [44]. In these papers, authors improved chemophotometric predictions, accounting for six stellar populations, from star metallicities 0.0004 to 0.05 ; a stellar population entails, by definition, all star clusters (i.e., particles) born with the same chemical compositions.

Each simulation self-consistently provides morphological, dynamic, and photometric evolution.

The initial mass function (IMF) is of Salpeter type [45] with upper and lower mass limits 100 and $0.01 \mathrm{M}_{\odot}$, respectively, as in Curir and Mazzei [44] and MC03.

All the model parameters have been tuned in these previous papers that analyzed the evolution of isolated collapsing triaxial halos initially composed of DM and gas. In these papers, the role of the initial spin of the halos and their total mass and gas fraction, as well as different IMFs, particle resolutions, SF efficiencies, and values of the feedback parameter, were all examined. The integrated properties of simulated galaxies, stopped at $15 \mathrm{Gyr}$, that is, their colors, 
TABLE 2: Input parameters of SPH simulation of UGC 7639.

\begin{tabular}{ccccccccc}
\hline$N_{\text {part }}(t=0)$ & $\begin{array}{c}a \\
{[\mathrm{kpc}]}\end{array}$ & $p / a$ & $\begin{array}{c}r_{1} \\
{[\mathrm{kpc}]}\end{array}$ & $\begin{array}{c}r_{2} \\
{[\mathrm{kpc}]}\end{array}$ & $\begin{array}{c}v_{1} \\
{[\mathrm{~km} / \mathrm{s}]}\end{array}$ & $\begin{array}{c}v_{2} \\
{[\mathrm{~km} / \mathrm{s}]}\end{array}$ & $\begin{array}{c}M_{1} \\
10^{10} \mathrm{M}_{\odot}\end{array}$ \\
\hline $10^{5}$ & 597 & $1 / 4$ & 117 & 549 & 12 & 48 & 40 \\
\hline
\end{tabular}

Notes. The columns are as follows: (1) total number of particles; (2) length of the semimajor axis of the primary halo; (3) pericentric separation of the halos in units of semimajor axis in col. (2); (4) and (5) distances of the halo centers of mass from the centre of mass of the global system; (6) and (7) velocity moduli of the halo centers in the same frame; (8) and (9) total masses of each system.

absolute magnitudes, metallicities, and mass to light ratios, had been successfully compared with those of local galaxies ([44], Figure 17; [38, 46], Figure 8). In particular, a slightly higher SFR, compared with the other possibilities examined in MC03, arises from our IMF choice (see MC03: Figure 1); this allows for the lowest feedback strength (63\% less than that in the same simulation with lower mass limit $0.1 \mathrm{M}_{\odot}$ ) and for the expected rotational support when disk galaxies are formed (MC03).

As pointed out by Kroupa [47], this slope is almost the same as the universal mass function that links the IMF of galaxies and stars to those of brown dwarfs, planets, and small bodies (meteoroids and asteroids [48]).

We point out that the SFR, which drives the evolution of the global properties of the simulated galaxies, converges when the initial particle number is above $10^{4}$ (see MC03 for a discussion and their Figure $1[49,50])$.

From our grid of SPH simulations, we singled out those that simultaneously account (i.e., at the same snapshot) at least for the following three observational constraints, which correspond to the global properties of UGC 7639:

(1) total B-band absolute magnitude within the range allowed from observations (Section 2.1);

(2) best fit of the integrated observed SED;

(3) morphology matching the observed one in the same bands and with the same spatial scale ( $\operatorname{arcsec} / \mathrm{kpc})$.

We direct the interested reader to the papers by Mazzei et al. [35], where our approach has been applied to early-type galaxies of two groups, USGC 376 and LGG 225, and Mazzei et al. [36], where our approach has been exploited to match not only photometric, but also structural (e.g., disk versus bulge) and kinematical (gas versus stars) properties of two S0 galaxies, namely, NGC 3626 and NGC 1533, to predict their evolution.

The initial conditions of the simulation selected correspond to an encounter: these conditions are shown in Table 2. The initial gas fraction is 0.11 in both systems. This value is very similar to the value of 0.13 found by Gonzalez et al. [51] by analyzing a large sample of clusters with different total masses. The initial gas mass resolution is $10^{6} \mathrm{M}_{\odot}$, while that of DM particles is nine times larger. The gravitational softening is $1,0.5$, and $0.05 \mathrm{kpc}$ for DM, gas, and star particles, respectively. The final number of particles at least doubles the initial number in Table 2.

The results presented in the next sections are the predictions of the simulation which best reproduces all the previous conditions (points 1-3) at the same snapshot. This snapshot sets the age of the simulation and the age of the galaxy. This means that the onset of the star formation is delayed compared to the beginning of the simulation, so that the age of the simulation is higher than the galaxy age.

\subsection{Results}

3.1.1. Comparison with Observations. The global properties of UGC 7639, that is, its total B-band absolute magnitude, integrated SED, and morphology, are well matched by a simulation corresponding to an encounter, not a merger, of two systems with mass ratio $4: 1$ and total mass $5 \times 10^{11} \mathrm{M}_{\odot}$ (Table 2); UGC 7639 corresponds to the less massive system.

The age of the galaxy at the snapshot which best fits the global properties of UGC 7639 is $8.6 \mathrm{Gyr}$, in good agreement with age estimates from synthetic CMD comparisons of Tolstoy et al. [4] for DGs in LV. This corresponds to a total B-band absolute magnitude of $-15.6 \mathrm{mag}$, in good agreement with the data (Section 1 and Table 1). At this time our target lies about $0.5 \mathrm{Mpc}$ away from the primary system, and the two galaxies are connected by a thin bridge of cold gas. We point out that this relative distance is greater than any projected distance on the sky plane. Karachentsev et al. [14] report NGC 4258 as the main disturber, that is, the neighboring galaxy producing the maximal tidal influence on UGC 7639. In contrast, from our analysis, the Canes Venatici member whose total B-band magnitude agrees at best with our predictions turns out to be NGC 4242, with $M_{B} \cong-19$ mag (HyperLeda, [52]), at a projected distance of $0.4 \mathrm{Mpc}$.

Figure 4 compares, on the same spatial scale and photometric band, the morphologies of our luminosity density map at the best-fit snapshot with the observed one. Figure 5(b) compares the r-band luminosity profile measured in Section 2.1 (blue circles) with that extracted from our simulated r-band luminosity density map (solid line) in Figure 4(a). The model profile has been derived with the same method (i.e., using IRAF ellipse routine) used to obtain the observed profile (see Section 2.1) and the same spatial resolution.

Figure 5(a) shows the comparison between the observed SED and our predictions. The amount of cold gas (i.e., $T<$ $20,000 \mathrm{~K}$ ) expected inside the simulated map in Figure 4 is about $8 \times 10^{7} \mathrm{M}_{\odot}$. Since the cooling time of this gas is much shorter than the snapshot time range $(0.151 \mathrm{Gyr}$, Section 3$)$, this amount represents the upper limit of the mass of cold gas, thus in agreement with the value derived from the Tully catalog (see Section 2.2). 


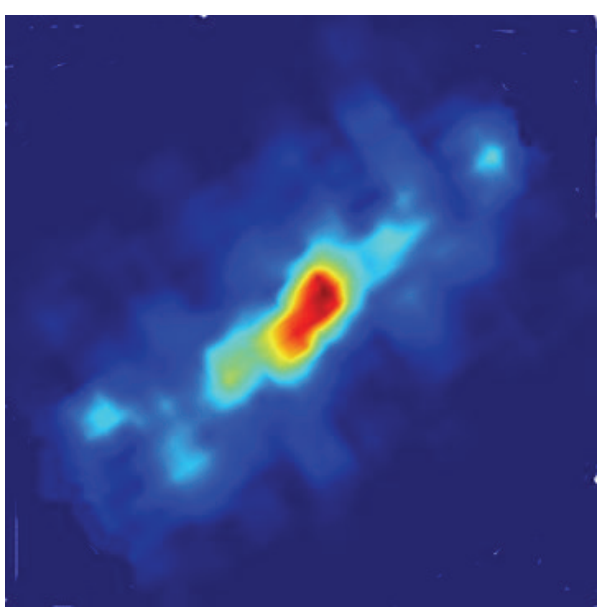

(a)

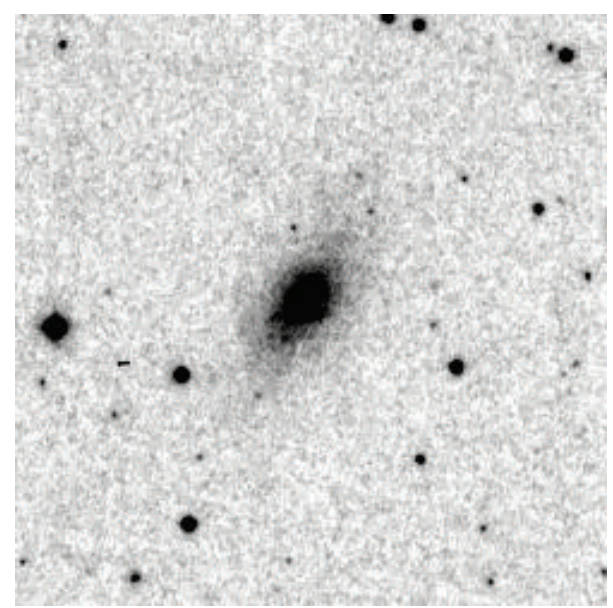

(b)

FIGURE 4: Morphology of UGC 7639. (a) r-band luminosity density map from our simulation at the best-fitting snapshot (see text), on the same scale as the observed one. (b) $7^{\prime} \times 7^{\prime}$ r-band image (dss1). (a) is normalized to its total flux.

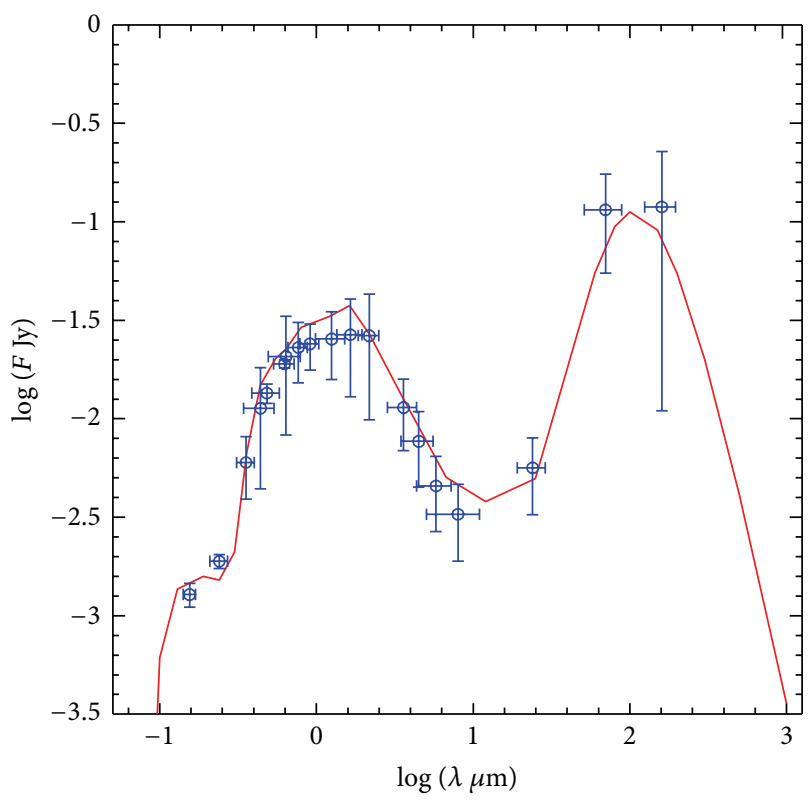

(a)

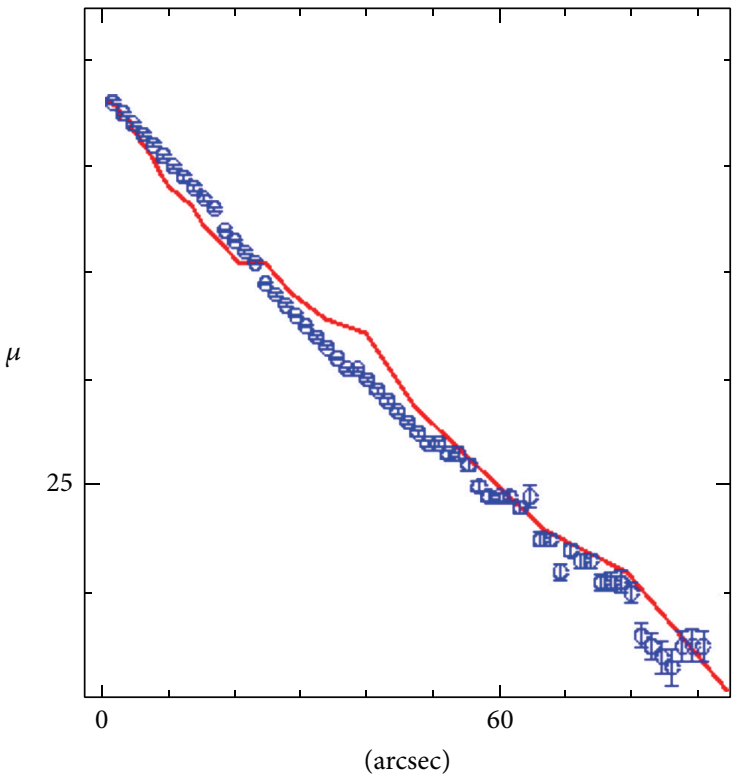

(b)

FIGURE 5: (a) the SED of UGC 7639. Blue filled circles correspond to UV/Optical/IR/FIR data, in particular FUV and NUV total fluxes from Table 1, u, g, r, i, and z, fluxes from NASA-Sloan Atlas [29], B and R from Bremnes et al. [20], and IR and FIR from Dale et al. [53]. Error bars account for bandwidth and $3 \sigma$ flux uncertainties. The solid (red) line shows the prediction of our model (see text). (b) the r-band luminosity profile of our simulated image in Figure 4 is compared with that measured in the same band and shown in Figure 2. Error bars $<1 \%$ do not appear in the figure.

The predicted FIR SED is composed by a warm and a cold dust component, both including polycyclic aromatic molecules, as described by Mazzei et al. [40, 41]. Warm dust is located in a region of high radiation, that is, in the neighborhood of OB stars (HII regions), whereas cold dust is heated by the interstellar diffuse radiation field. The distribution of diffuse radiation field best fitting the FIR-SED is the same as described by Mazzei et al. [40], that is, a disk of gas and stars, in agreement with our findings in Section 2.1. The intensity field of such component is four times higher than the average in our own galaxy, with a warm/cold energy ratio of 0.3 . It follows that the contribution of the warm component to the dust luminosity, $L_{\mathrm{FIR}}$ of UGC 7639, is about $25 \%$, like that of our own galaxy [40]. A more detailed FIR coverage is required to derive strong conclusions concerning these points; however the greater intensity of the diffuse radiation field we derive agrees with the lower metallicity of stars in this dwarf compared with that in our galaxy. It is well known that low metallicity stars are more luminous than stars with solar composition (see [54], for a review). 


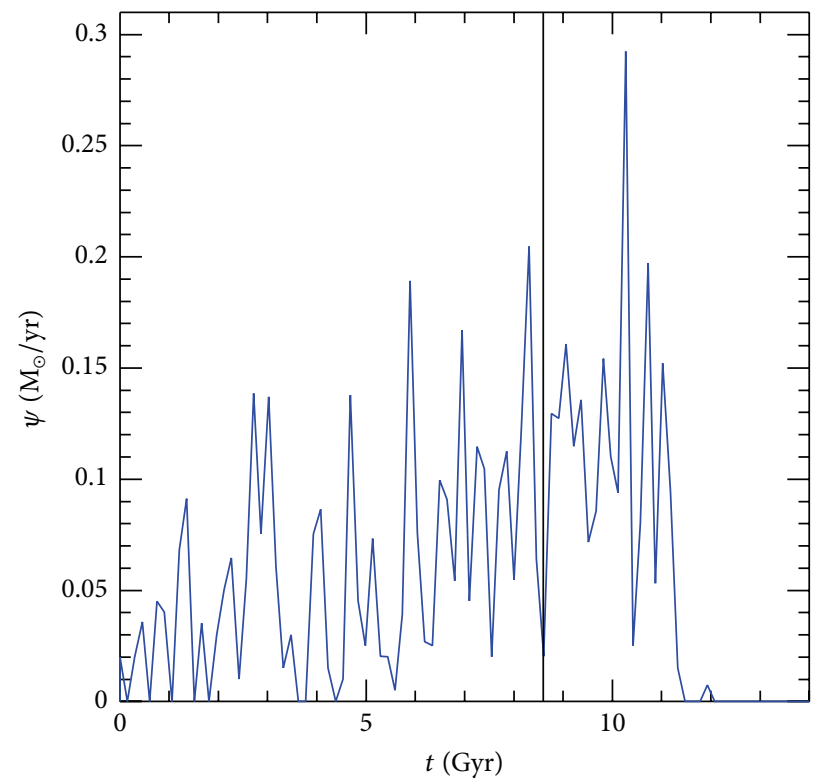

(a)

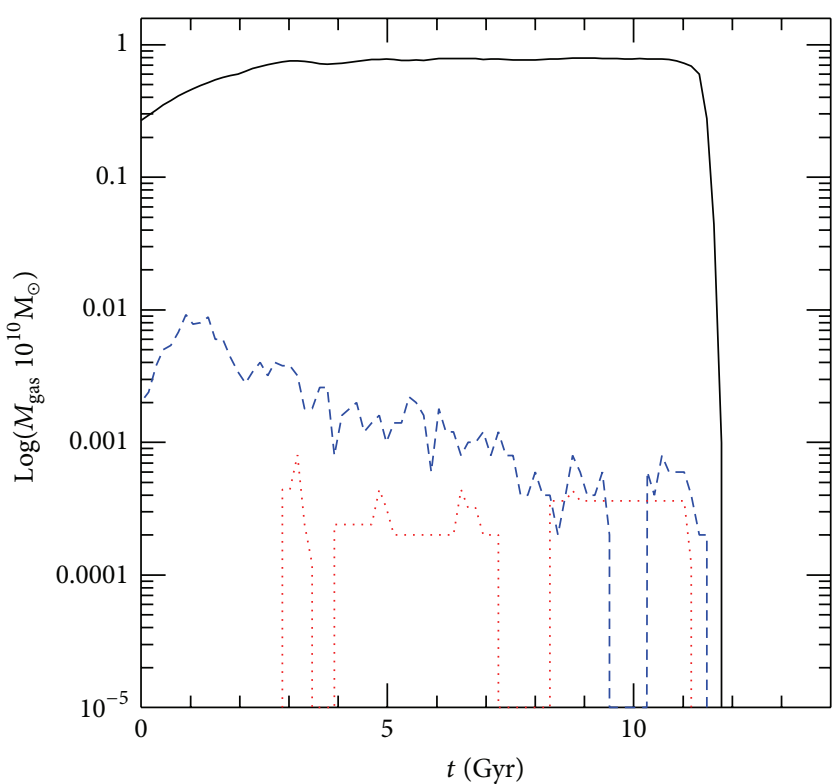

(b)

FIGURE 6: (a) The evolution of the SFR predicted by our simulation for UGC 7639. The black vertical line corresponds to the galaxy age (8.6 Gyr; see text). (b) Gas accretion history (solid line), that is, the evolution of the gas mass inside a radius of $50 \mathrm{kpc}$ on the r-band image luminous center of the galaxy from our selected SPH simulations. The (blue) dashed and the (red) dotted lines correspond to the gas with temperatures $10^{4} \mathrm{~K}$ and $10^{6} \mathrm{~K}$, respectively.

The average star metallicity provided by the best-fit snapshot for this DG is indeed 10-20 times lower than that in the solar neighbor, that is, about 0.001 . There are no measures of star metallicity available for this dwarf; however DGs are known as low-metallicity galaxies (e.g., [55]).

The current SFR at the selected snapshot is $\cong 0.03 \mathrm{M}_{\odot} \mathrm{yr}^{-1}$, in good agreement with UV-based estimates (see Section 2.2) accounting for a factor of 2.4 due to the lower mass limit of the IMF in our simulation. The average age of galaxy population within $R_{25} \simeq 2 \mathrm{kpc}$, weighted by B-band luminosity, is almost $1 \mathrm{Gyr}$, and the total mass inside the same radius is $5.3 \times$ $10^{6} \mathrm{M}_{\odot}$, with a ratio between DM and gas+stars mass of about 10. The total mass of stars in Figure 4 is $1.9 \times 10^{8} \mathrm{M}_{\odot}$ and the B-band $\mathrm{M} / \mathrm{L}$ ratio results to be $4 \mathrm{M}_{\odot} / \mathrm{L}_{\odot}$.

3.1.2. Evolution. Figure 6(a) shows the behavior of the SFR that drives the evolution of UGC 7639. We want to highlight that the age of the simulation corresponding to our best-fit snapshot is $12.5 \mathrm{Gyr}$, since in the $10^{11} \mathrm{M}_{\odot}$ halo of UGC 7639 the star formation starts about $\approx 4 \mathrm{Gyr}$ after the beginning of the simulation. After its onset, the SFR increases, on average, up to a galaxy age of $10.3 \mathrm{Gyr}$ (Figure 6) when it reaches its maximum value, 10 times higher than that at the best-fit snapshot (8.6 Gyr, see above). Pacifici et al. [56] found that low-mass galaxies have, on average, a rising star formation history, in good agreement with our predictions. Moreover, it is well known (e.g., [57]) that low-mass galaxies, especially dwarfs, are susceptible to episodic (bursty) star formation events. This behavior is also well reproduced by our simulation. Note that the burst duration estimate is affected by time resolution and burst definition. Time resolution is
$0.151 \mathrm{Gyr}$ for our best-fit simulation (Section 3). However, $302 \mathrm{Myr}$ before the best-fit snapshot the SFR is about 10 times higher than its present-day value (see Section 3.1.1). Moreover, according to its oscillating behavior of Figure 6(a), the time range from a maximum to a minimum value of the SFR is, on average, $604 \mathrm{Myr}$, in good agreement with the average estimate by McQuinn et al. [58].

Looking at the latest stages of the evolution, that is, the remaining $4 \mathrm{Gyr}$ before stopping our run, we noted that the burst is quenched after the gas is both exhausted and gradually removed (Figure 6(b)), while our dwarf is approaching more and more to the massive companion.

The behavior of the SFR cannot be provided by a simulation of the same halo evolving in isolation, as shown in Figure 1 of MC03. In that case, the SFR neither increases on average nor fades at the latest stage of the evolution.

The fate of our DG is thus to resemble that of a dwarf elliptical galaxy, devoid of gas and with red colors given the lack of young stars (Figure 6). The path predicted in the rest-frame UV color-magnitude diagram (CMD) also highlights this fate (Figure 7). The galaxy lies in a region corresponding to the extension of Wyder et al. [25] blue sequence (BS, cyan line in Figure 7) toward lower r-band luminosities. The BS is traced by the local spiral and starburst galaxies. By comparing Figure 7 with Figure 6(a), we note that our DG reaches its brightest r-band luminosity after $10.3 \mathrm{Gyr}$, that is, when its SFR gets its maximum value. Figure 6(b) shows that the SFR fades as the gas fueling decreases. The active phase of our dwarf lasts about $11 \mathrm{Gyr}$. Then, the galaxy crosses the green valley and reaches the red sequence (magenta line in Figure 7), the locus of early-type 


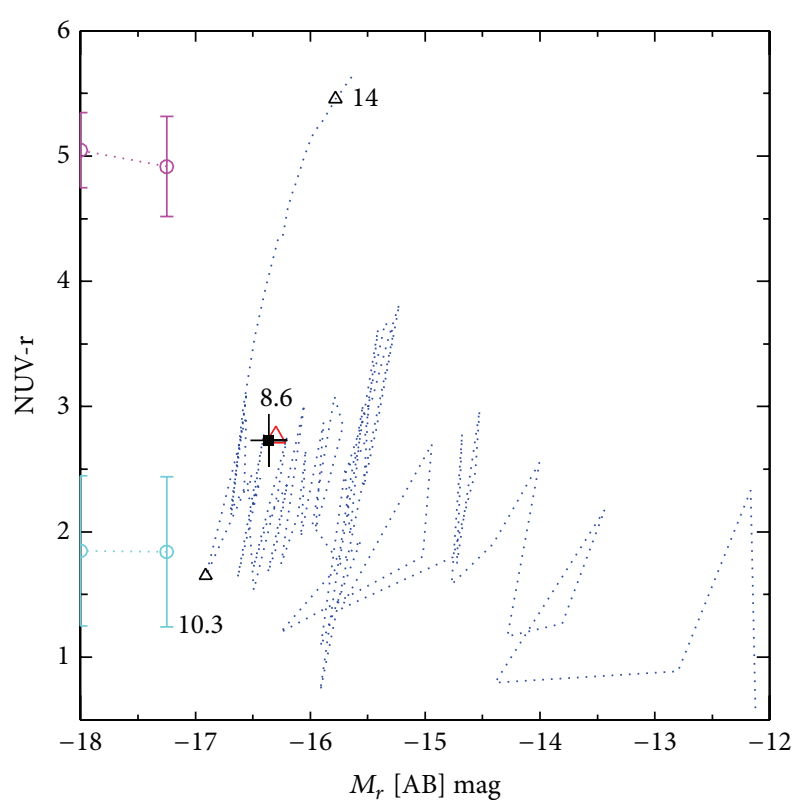

FIgURE 7: The rest-frame evolution of (NUV-r) versus $M_{r}$ colormagnitude diagram from our simulation of UGC 7639. Dotted line shows its evolutionary path; open symbols mark some meaningful evolutionary times in Gyr (see text); (red) triangle shows its current position. The observed position is given by the filled square, accounting for the same distance modulus as in Figure 4, and the corresponding $1 \sigma$ uncertainty. The red (magenta) and the blue (cyan) sequences are also plotted following the same prescriptions as in Figure 3.

galaxies, in two Gyrs, where it remains until the end of our simulation.

\section{Summary and Conclusions}

We used archive multiwavelength data together with SPH simulations with chemo photometric implementation to get insight into the present and evolutionary properties of the late-type dwarf UGC 7639 galaxy, a nearby DG hosting an inner star forming stellar population. We also investigated the UV-optical CMD of 22 galaxies in the Canes Venatici Cloud I, to which our target belongs, finding that UGC 7639 is among the faintest members, with the lowest cold gas amount.

We find that its global properties, namely, its total absolute B-band magnitude, whole SED, morphology, cold gas amount, and SFR, are well matched by an encounter with an object four times more massive. According to our data, the Canes Venatici member whose total B-band magnitude, $M_{B} \cong-19$ mag, agrees at best with our predictions turns out to be NGC 4242, which lies at a projected distance of $0.4 \mathrm{Mpc}$, in agreement with the simulation predictions.

The age of UGC 7639 we derived is $8.6 \mathrm{Gyr}$, whereas the average age of its stellar populations within $R_{25} \simeq$ $2 \mathrm{kpc}$, weighted by B-band luminosity, is $\approx 1 \mathrm{Gyr}$. Moreover, according to our simulation, its star formation will extinguish within $1.6 \mathrm{Gyr}$, thus likely leaving a dwarf elliptical galaxy.

As such, UGC 7639 is an example of DG whose global properties are well matched by our multiwavelength and multitechnique approach that seems to be very promising to explore the evolution of this class of galaxies (see the papers by Mazzei et al. [35, 36], devoted to analyze evolution of early-type galaxies). We will exploit our approach further in future papers to highlight, in a fully consistent way, both the evolution of this couple of interacting galaxies and that of other DGs.

\section{Conflict of Interests}

The authors declare that there is no conflict of interests regarding the publication of this paper.

\section{Acknowledgments}

Funding for the SDSS and SDSS-II has been provided by the Alfred P. Sloan Foundation, the Participating Institutions, the National Science Foundation, the U.S. Department of Energy, the National Aeronautics and Space Administration, the Japanese Monbukagakusho, the Max Planck Society, and the Higher Education Funding Council for England. The SDSS website is http://www.sdss.org/. The Astrophysical Research Consortium manages the SDSS for the participating institutions. The participating institutions are the American Museum of Natural History, Astrophysical Institute Potsdam, University of Basel, University of Cambridge, Case Western Reserve University, University of Chicago, Drexel University, Fermilab, the Institute for Advanced Study, the Japan Participation Group, Johns Hopkins University, the Joint Institute for Nuclear Astrophysics, the Kavli Institute for Particle Astrophysics and Cosmology, the Korean Scientist Group, the Chinese Academy of Sciences (LAMOST), Los Alamos National Laboratory, the Max-Planck-Institute for Astronomy (MPIA), the Max-Planck-Institute for Astrophysics (MPA), New Mexico State University, Ohio State University, University of Pittsburgh, University of Portsmouth, Princeton University, the United States Naval Observatory, and the University of Washington. This research has made use of the HyperLeda (http://leda.univ-lyonl.fr/; [59]) and the NASA/IPAC Extragalactic Database (NED), which is operated by the Jet Propulsion Laboratory, California Institute of Technology, under contract with the National Aeronautics and Space Administration.

\section{References}

[1] G. Gilmore, M. Wilkinson, J. Kleyna et al., "Observed Properties of Dark Matter: dynamical studies of dSph galaxies," Nuclear Physics B: Proceedings Supplements, vol. 173, pp. 15-18, 2007.

[2] F. Governato, C. Brook, L. Mayer et al., "Bulgeless dwarf galaxies and dark matter cores from supernova-driven outflows," Nature, vol. 463, no. 7278, pp. 203-206, 2010.

[3] J. J. Adams, J. D. Simon, M. H. Fabricius et al., "Dwarf galaxy dark matter density profiles inferred from stellar and gas kinematics," The Astrophysical Journal, vol. 789, no. 1, article 63, 2014.

[4] E. Tolstoy, V. Hill, and M. Tosi, "Star-Formation Histories, Abundances, and kinematics of dwarf galaxies in the local group," Annual Review of Astronomy and Astrophysics, vol. 47, pp. 371-425, 2009. 
[5] D. Crnojević, E. K. Grebel, and A. A. Cole, "A close look at the Centaurus A group of galaxies. III. Recent star formation histories of late-type dwarfs around M 83," Astronomy \& Astrophysics, vol. 530, article A59, 2011.

[6] N. Trentham, R. B. Tully, and A. Mahdavi, "Dwarf galaxies in the dynamically evolved NGC 1407 Group," Monthly Notices of the Royal Astronomical Society, vol. 369, no. 3, pp. 1375-1391, 2006.

[7] M. E. Sharina, I. D. Karachentsev, A. E. Dolphin et al., "Photometric properties of the Local Volume dwarf galaxies," Monthly Notices of the Royal Astronomical Society, vol. 384, no. 4, pp. 1544-1562, 2008.

[8] D. R. Weisz, E. D. Skillman, J. M. Cannon et al., "The recent star formation histories of M81 group dwarf irregular galaxies," Astrophysical Journal, vol. 689, no. 1, pp. 160-183, 2008.

[9] A. Bouchard, G. S. Da Costa, and H. Jerjen, "The Environmental influence on the evolution of local galaxies," Astronomical Journal, vol. 137, no. 2, pp. 3038-3052, 2009.

[10] E. K. Grebel, "Star formation histories of nearby Dwarf galaxies," Astrophysics and Space Science, vol. 277, supplement 1, pp. 231-239, 2001.

[11] V. Lora, A. Raga, and E. Grebel, "Interaction between IGM and a dwarf galaxy," Revista Mexicana de Astronomia y Astrofisica, vol. 51, p. 39, 2015.

[12] I. Saviane, V. D. Ivanov, E. V. Held et al., "Luminosity-metallicity relation for dIrr galaxies in the near-infrared," Astronomy and Astrophysics, vol. 487, no. 3, pp. 901-920, 2008.

[13] R. Rekola, H. Jerjen, and C. Flynn, "New distances of unresolved dwarf elliptical galaxies in the vicinity of the Local Group," Astronomy and Astrophysics, vol. 437, no. 3, pp. 823-835, 2005.

[14] I. D. Karachentsev, D. I. Makarov, and E. I. Kaisina, "Updated nearby galaxy catalog," The Astronomical Journal, vol. 145, no. 4, article 101, 2013.

[15] R. B. Tully, Nearby Galaxy Catalog, Cambridge University Press, Cambridge, UK, 1988.

[16] D. Makarov, L. Makarova, and R. Uklein, "Distances to dwarf galaxies of the Canes Venatici I cloud," Astrophysical Bulletin, vol. 68 , no. 2, pp. 125-138, 2013.

[17] P. Morrissey, T. Conrow, T. Barlow et al., "The calibration and data products of GALEX," The Astrophysical Journal Supplement Series, vol. 173, no. 2, pp. 682-697, 2007.

[18] M. Koleva, S. De Rijcke, W. W. Zeilinger, R. Verbeke, J. Schroyen, and L. Vermeylen, "On the origin of bursts in blue compact dwarf galaxies: clues from kinematics and stellar populations," Monthly Notices of the Royal Astronomical Society, vol. 441, no. 1, pp. 452-469, 2014.

[19] G. deVaucouleurs, G. de Vaucouleurs, A. de Vaucouleurs et al., Third Reference Catalogue of Bright Galaxies, Springer, Berlin, Germany, 1991.

[20] T. Bremnes, B. Binggeli, and P. Prugniel, "Structure and stellar content of dwarf galaxies: IV. B and R photometry of dwarf galaxies in the CVnI cloud," Astronomy and Astrophysics Supplement Series, vol. 141, no. 2, pp. 211-220, 2000.

[21] B. R. Parodi and B. Binggeli, "Distribution of star-forming complexes in dwarf irregular galaxies," Astronomy and Astrophysics, vol. 398, no. 2, pp. 501-515, 2003.

[22] S. S. Kaisin and I. D. Karachentsev, "Canes Venatici i cloud of galaxies seen in the $\mathrm{H} \alpha$ line," Astronomy and Astrophysics, vol. 479, no. 2, pp. 603-624, 2008.

[23] K. N. Abazajian, J. K. Adelman-McCarthy, M. A. Agüeros et al., "The seventh data release of the Sloan Digital Sky survey," The Astrophysical Journal, vol. 182, no. 2, pp. 543-558, 2009.
[24] R. I. Jedrzejewski, "CCD surface photometry of elliptical galaxies-I. Observations, reduction and results," Monthly Notices of the Royal Astronomical Society, vol. 226, no. 4, pp. 747$768,1987$.

[25] T. Wyder, K. Martin, D. Schiminovich et al., "The UV-optical galaxy color-magnitude diagram. I. Basic properties," The Astrophysical Journal Supplement Series, vol. 173, article 293, 2007.

[26] D. A. Hunter and B. G. Elmegreen, "Broadband imaging of a large sample of irregular galaxies," The Astrophysical Journal Supplement Series, vol. 162, no. 1, pp. 49-79, 2006.

[27] M. Fukugita, T. Ichikawa, J. E. Gunn, M. Doi, K. Shimasaku, and D. P. Schneider, "The sloan Digital Sky Survey photometric system," Astronomical Journal, vol. 111, no. 4, pp. 1748-1756, 1996.

[28] J. Lee, A. Gil de Paz, R. Kennicutt et al., "A GALEX ultraviolet imaging survey of galaxies in the local volume," The Astrophysical Journal Supplement Series, vol. 192, no. 1, article 6, 2011.

[29] M. R. Blanton, E. Kazin, D. Muna, B. A. Weaver, and A. Price-Whelan, "Improved background subtraction for the sloan digital sky survey images," The Astronomical Journal, vol. 142, no. 1, article 31, 2011.

[30] D. C. Martin, T. K. Wyder, D. Schimiovich et al., "The UVoptical galaxy color-magnitude diagram. III. Constraints on evolution from the blue to the red sequence," The Astrophysical Journal Supplement Series, vol. 173, no. 2, pp. 342-356, 2007.

[31] J. J. Fang, S. M. Faber, S. Salim, G. J. Graves, and R. M. Rich, "The slow death (o rebirth?) of extended star formation in $\mathrm{z} \sim$ 0.1 green valley early-type galaxies," The Astrophysical Journal, vol. 761, no. 1, article 23, 16 pages, 2012.

[32] R. C. Kennicutt Jr., "Star formation in galaxies along the Hubble sequence," Annual Review of Astronomy and Astrophysics, vol. 36, no. 1, pp. 189-231, 1998.

[33] I. D. Karachentsev and E. I. Kaisina, "Star formation properties in the local volume galaxies via $\mathrm{H} \alpha$ and far-ultraviolet fluxes," The Astronomical Journal, vol. 146, no. 3, article 46, 2013.

[34] C. M. Springob, M. P. Haynes, R. Giovanelli, and B. R. Kent, "A digital archive of H I 21 centimeter line spectra of optically targeted galaxies," The Astrophysical Journal, Supplement Series, vol. 160, no. 1, pp. 149-162, 2005.

[35] P. Mazzei, A. Marino, and R. Rampazzo, "The $N U V-r$ versus $M_{r}$ plane as a tracer of early-type galaxy evolution in the USGC U376 and LGG 225 groups," The Astrophysical Journal, vol. 782, no. 1, p. 53, 2014.

[36] P. Mazzei, A. Marino, R. Rampazzo, G. Galletta, and D. Bettoni, "Catching spiral-S0 transition in groups. Insights from SPH simulations with chemo-hotometric implementation," Advances in Space Research, vol. 53, no. 6, pp. 950-962, 2014.

[37] P. Mazzei and A. Curir, "Dark and luminous matter connections from smoothed particle hydrodynamic simulations of isolated triaxial collapsing systems," The Astrophysical Journal, vol. 591, no. 2, article 784, 2003.

[38] P. Mazzei, Recent Research Developments in A\&A, vol. 1, Research Signpost, Kerala, India, 2003.

[39] M. S. Warren, P. J. Quinn, J. K. Salmon, and W. H. Zurek, "Dark halos formed via dissipationless collapse. I-shapes and alignment of angular momentum," The Astrophysical Journal, vol. 399, no. 2, pp. 405-425, 1992.

[40] P. Mazzei, C. Xu, and G. de Zotti, "A model for the photometric evolution of disc galaxies from UV to far-IR," Astronomy \& Astrophysics, vol. 256, no. 1, pp. 45-55, 1992. 
[41] P. Mazzei, G. de Zotti, and C. Xu, "Models for the evolution of the spectral energy distribution of elliptical galaxies from ultraviolet to far-infrared wavelengths," Astrophysical Journal, vol. 422, no. 1, pp. 81-91, 1994.

[42] P. Mazzei, A. Curir, and C. Bonoli, "Self-consistent evolution of ring galaxies," The Astronomical Journal, vol. 110, no. 2, pp. 559572, 1995.

[43] C. J. Lonsdale, "The infrared background (theory)," in Proceedings of the STSI Symposium, D. Calzetti, M. Livio, and P. Madau, Eds., p. 145, Cambridge University Press, Baltimore, Md, USA, 1993.

[44] A. Curir and P. Mazzei, "SPH simulations of galaxy evolution including chemo-photometric predictions," New Astronomy, vol. 4, no. 1, pp. 1-20, 1999.

[45] E. E. Salpeter, "The luminosity function and stellar evolution," The Astrophysical Journal, vol. 121, p. 161, 1955.

[46] P. Mazzei, "Dark and luminous matter connections. Towards understanding galaxy evolution," http://arxiv.org/abs/astro-ph/ 0401509 .

[47] P. Kroupa, "The dark matter crisis: falsification of the current standard model of cosmology," Publications of the Astronomical Society of Australia, vol. 29, no. 4, pp. 395-433, 2012.

[48] B. Binggeli and T. Hascher, "Is there a universal mass function?" Publications of the Astronomical Society of the Pacific, vol. 119, no. 856, pp. 592-604, 2007.

[49] C. R. Christensen, T. Quinn, G. Stinson, J. Bellovary, and J. Wadsley, "Star formation and feedback in smoothed particle hydrodynamic simulations. II. Resolution effects," The Astrophysical Journal, vol. 717, no. 1, pp. 121-132, 2010.

[50] C. Christensen, T. Quinn, F. Governato et al., "Implementing molecular hydrogen in hydrodynamic simulations of galaxy formation," Monthly Notices of the Royal Astronomical Society, vol. 425, no. 4, pp. 3058-3076, 2012.

[51] A. H. Gonzalez, S. Sivanandam, A. I. Zabludoff, and D. Zaritsky, "Galaxy cluster baryon fractions revisited," Astrophysical Journal, vol. 778, no. 1, article 14, 2013.

[52] D. Makarov, P. Prugniel, N. Terekhova, H. Courtois, and I. Vauglin, "HyperLEDA. III. the catalogue of extragalactic distances," Astronomy and Astrophysics, vol. 570, article A5, 12 pages, 2014.

[53] D. Dale, S. Cohen, L. Johnson et al., "The Spitzer local volume legacy: survey description and infrared photometry," The Astrophysical Journal, vol. 703, pp. 517-556, 2009.

[54] C. Choisi, "Stellar nucleosinthesis and chemical evolution of galaxies," EAS Publication Series, vol. 27, pp. 25-39, 2007.

[55] C. A. Tremonti, T. M. Heckman, G. Kauffmann et al., "The origin of the mass-metallicity relation: insights from 53,000 starforming galaxies in the sloan digital sky survey," Astrophysical Journal Letters, vol. 613, no. 2, pp. 898-913, 2004.

[56] C. Pacifici, S. A. Kassin, B. Weiner, S. Charlot, and J. P. Gardner, "The rise and fall of the star formation histories of blue galaxies at redshifts $0.2<z<1.4$," The Astrophysical Journal Letters, vol. 762, no. 1, p. L15, 2013

[57] A. E. Bauer, A. M. Hopkins, M. Gunawardhana et al., "Galaxy And Mass Assembly (GAMA): linking star formation histories and stellar mass growth," Monthly Notices of the Royal Astronomical Society, vol. 434, no. 1, pp. 209-221, 2013.

[58] K. B. W. McQuinn, E. D. Skillman, J. M. Cannon et al., “The nature of starbursts. II. The duration of starbursts in dwarf galaxies?” Astrophysical Journal, vol. 724, no. 1, pp. 49-58, 2010.
[59] D. Makarov, P. Prugniel, N. Terekhova, H. Courtois, and I. Vauglin, "HyperLEDA. III. The catalogue of extragalactic distances," Astronomy \& Astrophysics, vol. 570, article A13, 2014. 

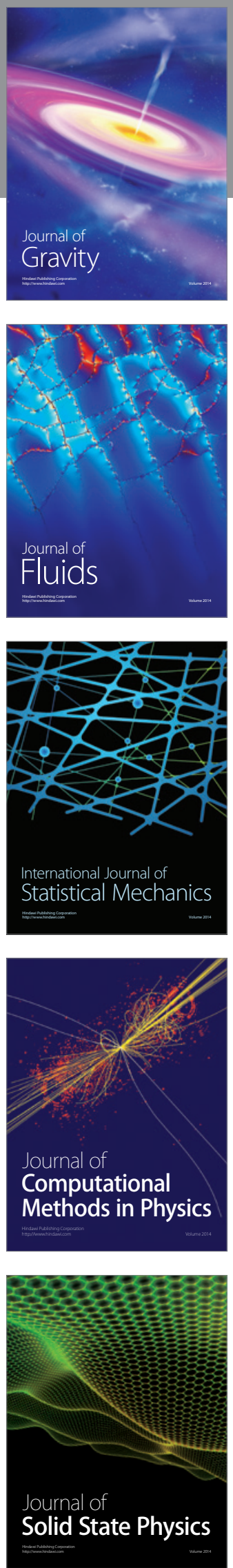

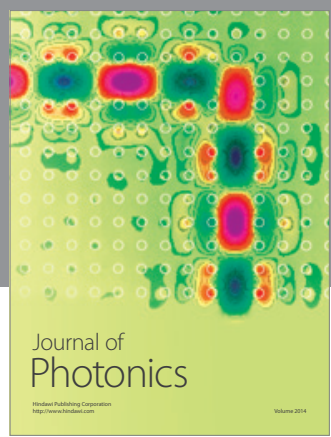

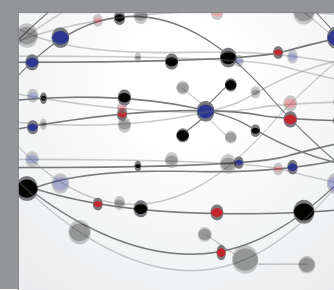

The Scientific World Journal

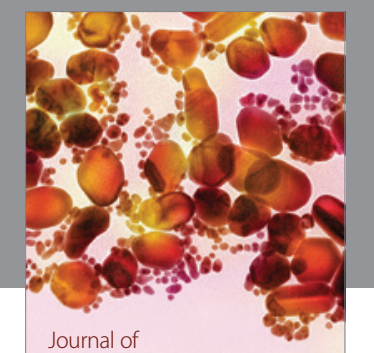

Soft Matter
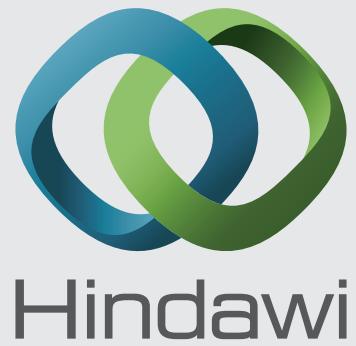

Submit your manuscripts at

http://www.hindawi.com
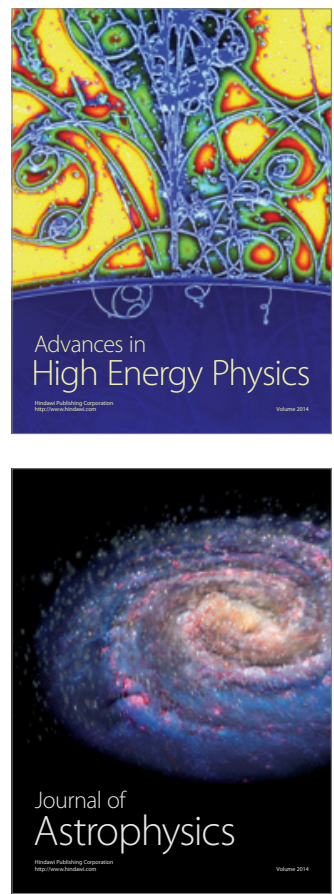
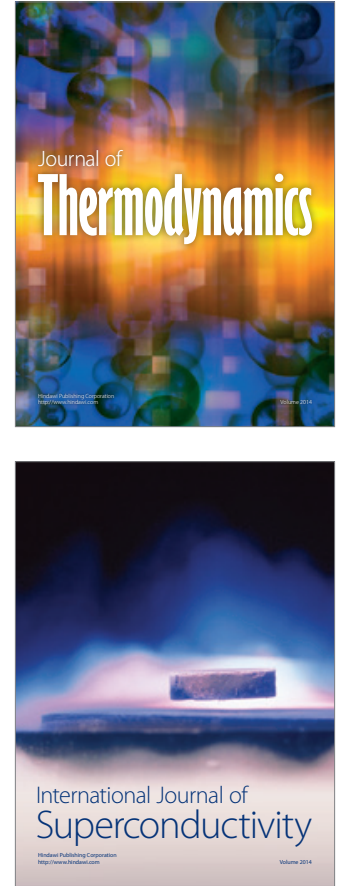
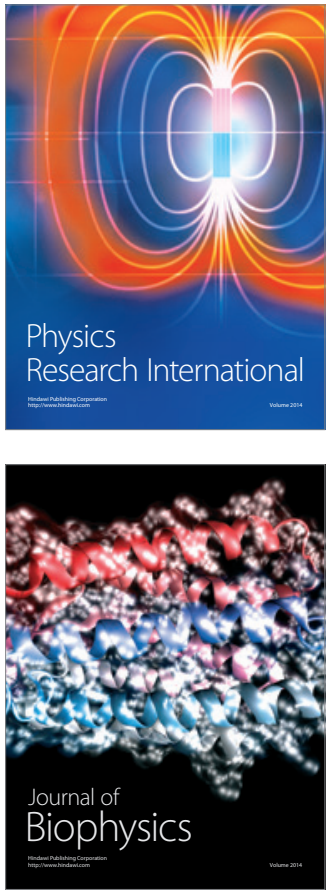
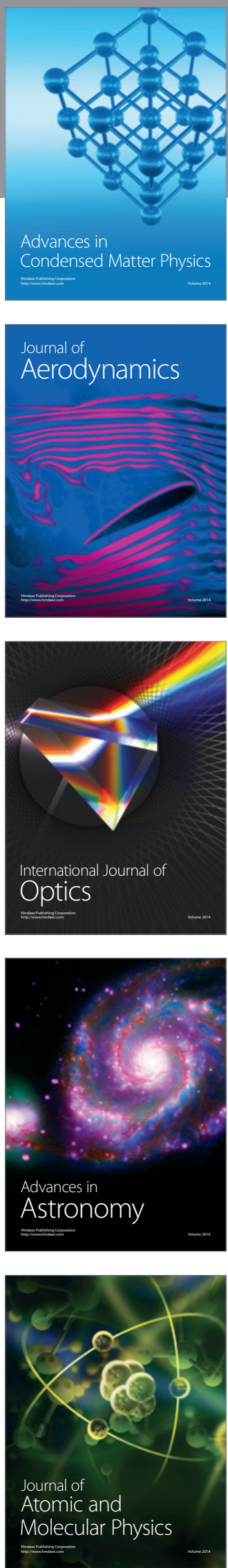